\title{
$p$-adic String Theories Provide Lattice Discretization to the Ordinary String Worldsheet
}

\author{
Debashis Ghoshal* \\ Harish-Chandra Research Institute, Chhatnag Road, Allahabad 211019, India
}

(Received 14 July 2006; published 9 October 2006)

\begin{abstract}
A class of models called $p$-adic strings is useful in understanding the tachyonic instability of string theory. These are found to be empirically related to the ordinary strings in the $p \rightarrow 1$ limit. We propose that these models provide discretization for the string worldsheet and argue that the limit is naturally thought of as a continuum limit in the sense of the renormalization group.
\end{abstract}

Although string theory is the leading candidate for a consistent quantum theory of gravity and enormous progress has been made in the subject, it is fair to say that the basic principles of the theory are not well understood. The standard computational tools of the theory give a limited understanding of nonperturbative issues, e.g., the tachyonic instabilities present in certain backgrounds of bosonic and superstring theories. Under these circumstances, simplified toy models can provide important insight. One such model is the $p$-adic string theory proposed in Ref. [1]. In these theories, defined for prime numbers $p$, the only excitation is the scalar field corresponding to the instability, for which all the amplitudes can be computed at the tree level [1-4]. Hence, the spacetime effective theory of the tachyonic scalar is known exactly. This allows one to analyze the instability through the process of tachyon condensation [5] and check for the validity of the conjectures by Sen [6].

Unexpectedly, the ordinary bosonic open string emerges in the $p \rightarrow 1$ limit [7], in which the effective action of the $p$ tachyon turns out to approximate that obtained from the boundary string field theory (BSFT) [8,9] of ordinary strings. BSFT was useful in proving the Sen conjectures $[7,10,11]$. This relation in the $p \rightarrow 1$ limit, however, is empirical. Moreover, strictly $p$ can take only discrete values. In this Letter, we consider the issue from the point of view of the string worldsheet. We advocate that the limit is to be understood in the sense of real space renormalization group (RG) transformation. We argue that (for any prime $p$ ) there is a sequence of string theories, each providing a lattice discretization to the ordinary worldsheet [the disk or the upper half-plane (UHP)] with a natural continuum limit in which the RG transformed effective value of $p$ tends to one. Thus, we find a physical interpretation of what were thought to be esoteric mathematical models of string theory. A preliminary version of these ideas was presented in the "12th Regional Conference on Mathematical Physics" held in Islamabad, Pakistan [12].

Recall the Koba-Nielsen formula for the tree-level scattering amplitude of $N$ on-shell open-string tachyons of momenta $k_{i}(i=1, \ldots, N)$ :

$$
\mathcal{A}_{N}=\int \prod_{i=4}^{N} d \xi_{i}\left|\xi_{i}\right|^{k_{1} \cdot k_{i}\left|1-\xi_{i}\right|^{k_{2} \cdot k_{i}}} \prod_{4 \leq i<j \leq N}\left|\xi_{i}-\xi_{j}\right|^{k_{i} \cdot k_{j}}
$$

where $k_{i}^{2}=2, \sum k_{i}=0$, the integrals are over the real numbers $\mathbf{R}$, and the integrand involves only absolute values. Except for $\mathcal{A}_{4}$, the integrals cannot be computed analytically. Faced with a similar situation, mathematicians often broaden the scope of the analysis by extending the problem to related structures. A close analog of $\mathbf{R}$ is the local field of $p$-adic numbers $\mathbf{Q}_{p}$. In order to describe this field, let us digress briefly.

We are familiar with the absolute value norm on the field of rational numbers $\mathbf{Q}$. However, it is possible to define other norms on $\mathbf{Q}$ consistently. To this end, fix a prime number $p$ and determine the highest powers $n_{1}$ and $n_{2}$ of $p$ that divides, respectively, the numerator $z_{1}$ and denominator $z_{2}$ in a rational number $z_{1} / z_{2},\left(z_{1}, z_{2}\right.$ coprime). The $p$-adic norm of $z_{1} / z_{2}$, defined as $\left|z_{1} / z_{2}\right|_{p}=p^{n_{2}-n_{1}}$, satisfies all the required properties, indeed even a stronger version, of the triangle inequality: $|x+y|_{p} \leq$ $\max \left(|x|_{p},|y|_{p}\right)$. Because of this last property, the distances are called ultrametric, a notion that is natural in measuring distances between generations or the multiple neardegenerate vacua of systems such as spin glasses [13].

If we complete $\mathbf{Q}$ by putting in the limit points of all Cauchy sequences, in which convergence is decided by the absolute value norm, we get $\mathbf{R}$. Instead, if we use the $p$-adic norm, we arrive at the field $\mathbf{Q}_{p}$. Any $p$-adic number $\xi \in \mathbf{Q}_{p}$ has a representation as a Laurent-like series in $p$ :

$$
\xi=p^{N}\left(\xi_{0}+\xi_{1} p+\xi_{2} p^{2}+\cdots\right)
$$

where $N \in \mathbf{Z}$ is an integer, $\xi_{n} \in\{0,1, \cdots, p-1\}, \xi_{0} \neq$ 0 , and $|\xi|_{p}=p^{-N}$. Details of materia $p$-adica are available in, e.g., Refs. [14-16]; some essential aspects are reviewed in Ref. [17].

Coming back to the amplitudes (1), Freund and Olson [1] modified these by replacing the absolute values by $p$-adic norms and the real integrals by integrals over $\mathbf{Q}_{p}$. These are, by definition, the amplitudes for the scattering of $N$ open $p$-string tachyons. The benefit is that all of these 
integrals over $\mathbf{Q}_{p}$ can be evaluated analytically. Equivalently, the tree-level effective action of the open $p$-string tachyon $T$ is known exactly [3,4]: In terms of a rescaled and shifted field $\varphi=1+g_{s} T / p$,

$$
\mathcal{L}_{p}=\frac{p^{2}}{g^{2}(p-1)}\left[-\frac{1}{2} \varphi p^{-(1 / 2) \square} \varphi+\frac{1}{p+1} \varphi^{p+1}\right] .
$$

We emphasize that, in the above, the boundary of the open $p$-string worldsheet is $p$-adic, but the spacetime in which the $p$-string propagates is the usual one. Once one arrives at the spacetime action (3), however, it can be extrapolated to all integers. Incidentally, there is also another extrapolation, unrelated to this, in which the Veneziano amplitude $\mathcal{A}_{4}$ (expressed in terms of the gamma function) is modified to be valued in $\mathbf{Q}_{p}$ [18-20].

The equation of motion from (3) admits the constant solutions $\varphi=1$ (unstable vacuum with the D-brane) and $\varphi=0$. There is no perturbative open-string excitation around the latter, and, hence, it is to be identified as the (meta)stable closed $p$-string vacuum. There are also soliton solutions. For any (spatial) direction, there is a localized Gaussian lump [3]. When identified as the different D- $m$-branes, the descent relations between these confirm the Sen conjectures [5].

If one substitutes $p=1+\epsilon$ in (3) and takes the limit $\epsilon \rightarrow 0$, one obtains [7] (see also the prescient comments in Ref. [21]), after a field redefinition $\varphi=e^{-T / 2}$, the effective action of the tachyon of the ordinary open-string theory calculated from BSFT $[8,9]$. In fact, after a noncommutative deformation of (3), the Gaussian soliton of $p$-string theory generalizes to a one-parameter family of exact solutions. In the limit $p \rightarrow 1$, one finds a solution to the ordinary string theory, for all values of the deformation parameter [22]. (The noncommutativity in ordinary strings comes from a constant $B$-field background. Attempts to find the worldsheet origin of it in $p$-string theory were made in Refs. [23,24].)

At first sight, the relation to the ordinary strings is all the more surprising and counterintuitive from the point of view of the $p$-string "worldsheet." In fact, the worldsheet itself, the boundary of which is $\mathbf{Q}_{p}$, is not in the least obvious $[25,26]$. At tree level, the analog of the unit disk or the UHP of the usual theory is an infinite lattice with no closed loops, i.e., a uniform tree $\mathcal{B}_{p}$ in which $p+1$ edges meet at each vertex (see Fig. 1). This is the familiar Bethe lattice $\mathcal{B}_{p}$, known in the context of $\mathbf{Q}_{p}$ as the Bruhat-Tits tree. Its boundary, defined as the union of all infinitely remote vertices, can be identified with $\mathbf{Q}_{p}$. To see this, one may use, e.g., the representation (2), in which case the integer $N$ chooses a branch along the dotted path (in Fig. 1) and the infinite set of coefficients $\xi_{n}$ determine the path to the boundary. On the other hand, the tree $\mathcal{B}_{p}$ is the (discrete) homogeneous space $\operatorname{PGL}\left(2, \mathbf{Q}_{p}\right) / \operatorname{PGL}\left(2, \mathbf{Z}_{p}\right)$. This construction parallels the case of the ordinary string theory.

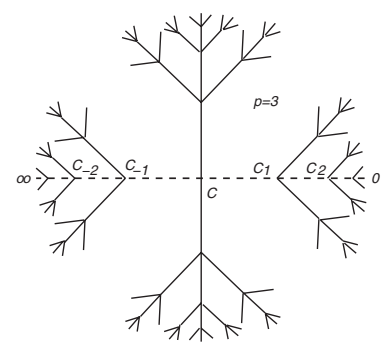

FIG. 1. The worldsheet of the 3-adic string $\mathcal{B}_{3}, \partial \mathcal{B}_{3}=\mathbf{Q}_{3}$. The dotted line is the path from the boundary points 0 to $\infty$.

The worldsheet action on $\mathcal{B}_{p}$ is the natural discrete lattice action for the free massless scalars $X^{\mu}$. The action of the Laplacian at a site $z \in \mathcal{B}_{p}$ is $\nabla^{2} X^{\mu}(z)=$ $\sum_{i} X^{\mu}\left(z_{i}\right)-(p+1) X^{\mu}(z)$, where $z_{i}$ are the $p+1$ nearest neighbors of $z$. It was shown in Ref. [25] that, starting with a finite Bethe lattice and inserting the tachyon vertex operators on the boundary, one recovers the prescription of Refs. [1,3] in the thermodynamic limit.

Naively, the lattice is one-dimensional for $p=1$. However, the relation to the ordinary string is through the limit $p \rightarrow 1$, and it is not apparent how to make sense of this for the discrete variable $p$. This is the problem we will address in the following. First, we claim that $\mathcal{B}_{p}$ gives a discretization of the disk or UHP. However, in $\mathcal{B}_{p}$, the number of sites up to some generation $n$ from an origin $C$ (say) grows exponentially for large $n$ :

$$
\mathcal{N}_{n} \sim \exp (n \ln p)
$$

Therefore, its formal dimension is infinite. Indeed, the mean field theory of model systems on Bethe lattices is exact.

The tacit assumption above is that the embedding is in an Euclidean space. On the other hand, in a $d$-dimensional hyperbolic space with the metric $d s_{H}^{2}=d r^{2}+$ $R_{0}^{2} \sinh ^{2}\left(r / R_{0}\right) d \Omega_{d-1}^{2}$, the volume of a ball of radius $R$ ( $R \gg R_{0}$, the radius of curvature) also grows exponentially for large $R$ :

$$
\operatorname{vol}_{d}(R) \sim \exp \left(\frac{d-1}{R_{0}} R\right) .
$$

This suggests a natural embedding of $\mathcal{B}_{p}$ in hyperbolic spaces. Parametrizing

$$
p=1+\frac{a}{R_{0}}(d-1)
$$

and considering the limit $a \rightarrow 0$ so that $p \rightarrow 1$, the formulas (4) and (5) agree for $\lim _{\substack{n \rightarrow \infty \\ a \rightarrow 0}} n a=R$, from which $a$ is seen as the lattice spacing. Thus, a uniform Bethe lattice $\mathcal{B}_{p}$ can be used to discretize a hyperbolic space of constant negative curvature. Moreover, $p \rightarrow 1$ provides a natural continuum limit. This is true, in particular, when the dimension $d=2$, the case of our interest. In fact, the embedding of $\mathcal{B}_{p}$ into the unit disk or UHP equipped with, say, 
the Poincare metric, is isometric. It is related to the hyperbolic tiling of the disk or UHP and often has an interesting connection with the fundamental domains of the modular functions of SL(2, C) and its subgroups [27].

The standard way to obtain a continuum limit from a lattice regularization is to go to lattices with smaller lattice spacings and eventually consider the limit in which this becomes vanishingly small. Suppose we start with the black sublattice in Fig. 2, the boundary of which is $\mathbf{Q}_{3}$. In comparing this to the full lattice, we see that between two neighboring black nodes there are two gray nodes, which in turn branch further so that the full lattice is similar to the black one. One can associate this process to a very precise mathematical construction related to $\mathbf{Q}_{p}$.

The $p$-adic field (like $\mathbf{R}$ ) is not closed algebraically. That is, not all roots of polynomials with coefficients in the field belong to it. For $\mathbf{R}$, one can adjoin a root of $x^{2}+$ $1=0$ and extend to the algebraically closed (and complete) field of complex numbers $\mathbf{C}$. It is said to be an index two extension; i.e., $\mathbf{C}$ is a two-dimensional vector space over $\mathbf{R}$. The story is more complex for $\mathbf{Q}_{p}$, for which there are infinitely many algebraic extensions none of which is closed. Now consider a finite extension $\overline{\mathbf{Q}}_{p}^{(n)}$ of index $n$. There are several such, and an integer $e$, called the ramification index, partially distinguishes between them. It turns out that $e$ divides $n$, so that $f=n / e$ is again an integer [14-16]. For simplicity, we consider first an extension for which $e=n$ (it is called totally ramified). The lattice associated with this extension can be obtained from the original Bethe lattice of $\mathbf{Q}_{p}$ through the process described in the last paragraph. Namely, start with the black lattice for $\mathbf{Q}_{p}$ and introduce $(e-1)$ new nodes between the existing ones. Connect (infinite) gray branches to these so that the tree is uniform with coordination number $p$ as before. In the other cases when $e<n$, one also needs to introduce an infinite number of new edges and nodes so that the resulting tree is uniform with coordination number $p^{f}[25,26]$.

In the extended field $\overline{\mathbf{Q}}_{p}^{(n)}$, there is a special element $\pi$, called the uniformizer, that plays the role of $p$ for $\mathbf{Q}_{p}$. Specifically, any element of $\overline{\mathbf{Q}}_{p}^{(n)}$ can be expressed as a Laurent series in terms of $\pi$ [just like (2)], and the norms of its elements are integer powers of $\pi$. In particular, for $p \in$

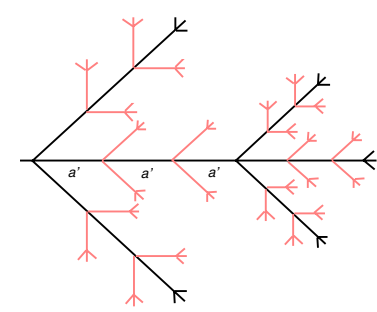

FIG. 2 (color online). The lattice with spacing $a^{\prime}$ leads to a coarse-grained one with spacing $a=m a^{\prime}$ ( $m=3$ here), when the gray branches are integrated out.
$\overline{\mathbf{Q}}_{p}^{(n)}:$

$$
p \simeq \pi^{e},
$$

where the approximate equality indicates the leading term in the expansion. Parametrizing both $p$ and $\pi$ as in (6), the lattice spacing $a^{\prime}$ of $\overline{\mathcal{B}}_{p}^{(n)}$ is related to $a$ of $\mathcal{B}_{p}$ as $a \simeq n a^{\prime}$, as is apparent from the construction. Thus, for larger and larger extensions, $\pi \simeq p^{1 / e}$ approaches the value 1 for any $p$. The corresponding lattices provide finer discretizations and a passage to the continuum limit.

The construction of the previous section suggests a way to understand the limit $p \rightarrow 1$ through a sequence of string theories based on the extensions of $\mathbf{Q}_{p}$. For simplicity, let us consider a totally ramified extension. Apparently, there is a puzzle, because the tachyon amplitudes (1) for this turn out to be exactly the same as those for $\mathbf{Q}_{p}$. This is because the coefficients in the Laurent expansions of both are from the same set; the lattices are similar; therefore, the measures that affect the integrals work out to be identical [3]. Hence, the effective actions of the tachyon of these two theories are identical. String theories based on extensions of $\mathbf{Q}_{p}$ were already considered in Refs. [1,3]; indeed, the very first paper on $p$-adic string theory [1] dealt with the quadratic extensions of $\mathbf{Q}_{p}$. In analogy with ordinary strings, it was thought to be a theory of closed strings. The theories based on higher extensions were called even more closed strings. In hindsight, it is natural to think of all of these as open strings.

Returning to the apparent paradox, the resolution comes from the following. In taking a continuum limit, one is not really interested in the results separately for the two theories but, rather, in comparing the degrees of freedom of the coarse-grained lattice from the fine one from the perspective of a (real space) RG. In order to do this, only the degrees of freedom on the gray nodes and branches (see Fig. 2) should be integrated out. This leaves one with the black sublattice with some effective interaction between these residual degrees of freedom. A rescaling of the lattice so that the spacing $a \rightarrow b a=a^{\prime}$ completes the RG transformation.

Let us see the effect of these on Green's function for the Laplace equation on the Bethe lattice. It is more transparent for the Dirichlet problem, for which we have [25]

$$
\mathcal{D}(z, w)=\frac{p}{p^{2}-1} p^{-d(z, w)},
$$

where $d(z, w)$ is the number of steps in lattice units between the sites $z$ and $w$. Since the spacing in $\mathcal{B}_{p}$ is $e=n$ times that in $\overline{\mathcal{B}}_{p}^{(n)}, d_{\mathcal{B}}=e d_{\overline{\mathcal{B}}^{(n)}} \equiv e \bar{d}$ and after integrating out the intermediate sites, $\mathcal{D}_{\text {eff }}=\left[p /\left(p^{2}-1\right)\right] p^{-e \bar{d}(z, w)}$. When the lattice is rescaled, the original form of the kernel is recovered with the substitution $p \rightarrow \pi=p^{1 / e}$. The Green's function $\mathcal{N}(z, w)$ for the Neumann problem is roughly the logarithm of $\mathcal{D}(z, w)$ [25], so the same argument holds there as well. Thus, the effect of the RG trans- 
formation on the tachyon action (3) is to replace $p \rightarrow \pi=$ $p^{1 / e}$. The action for the ordinary bosonic string is obtained in the limit $e \rightarrow \infty$, which is a continuum limit in the sense of RG.

The above argument can be straightforwardly extended to any finite extension of $\mathbf{Q}_{p}$. Let us also note that only the unramified extension $(e=1)$ is unique; there are several extensions differing in the details of the structure of the field. However, the associated lattices, which are the objects of interest to us, are specified only by the values of $e$ and $f$. It is not clear to us if the nonuniqueness has any role to play for the string theories based on these fields.

Further evidence comes from the problem of random walk on a Bethe lattice, for which Ref. [28] found an exact solution. This goes over to the solution of the Brownian motion on a hyperbolic space of constant negative curvature in the (formal) limit $p \rightarrow 1$. Thus, the Green's function for the diffusion equation on the hyperbolic disk or UHP can be obtained as a continuum limit from the Bethe lattice. The well known relation between the kernel of the diffusion equation and the Green's function of a free scalar field theory can be to used obtain the latter. We are interested in a diffeomorphism and Weyl invariant free scalar field theory coupled to the metric on the disk or UHP. There are also marked points corresponding to asymptotic states given by vertex operators on its boundary. Only hyperbolic metrics can be consistently defined on such a surface. Further, with the freedom from diffeomorphism and Weyl invariance, the metric can be made one of constant negative curvature. In the worldsheet functional integral, therefore, the contribution is from such a surface. The continuum limit of a scalar field theory on a Bethe lattice would seem to give a good approximation.

In summary, we have argued that the observation that the effective field theory of the tachyon of the $p$-adic string approximates that of the ordinary string in the $p \rightarrow 1$ limit can be understood in terms of RG flow on a sequence of open-string theories. Each of these theories provides a discretization of the tree-level worldsheet of the ordinary string and the $p \rightarrow 1$ limit is a continuum limit in the sense of (real space) RG. They also have a mathematically precise description in terms of algebraic extensions of increasing degree of the $p$-adic field.

A few brief closing remarks. First, the discretization here is quite different from the standard one in terms of large $N$ random matrices. However, the zeros of the partition function of the Ising and Potts models on random lattices from $1 \times 1$ matrices and on Bethe lattices are identical [29], suggesting some kind of complementarity between the two approaches. Second, the Bethe lattice worldsheet is isometric to the disk or UHP with a metric of constant negative curvature. This is a solution to the equation of motion of the Liouville field theory and is interpreted as the D0-brane [30]. Finally, the $p \rightarrow 1$ limit in terms of a sequence of theories based on lattices with smaller spacings may be useful in finding the "closed" strings of the $p$-adic theory.

It is a pleasure to thank Chandan Dalawat, Peter Freund, and Stefan Theisen for useful discussions. Hospitality at the Albert Einstein Institute, Germany, where a part of the work was done, is acknowledged gratefully.

*Electronic address: ghoshal@mri.ernet.in

[1] P. Freund and M. Olson, Phys. Lett. B 199, 186 (1987).

[2] P. Freund and E. Witten, Phys. Lett. B 199, 191 (1987).

[3] L. Brekke, P. Freund, M. Olson, and E. Witten, Nucl. Phys. B302, 365 (1988).

[4] P. Frampton and Y. Okada, Phys. Rev. D 37, 3077 (1988); Phys. Rev. Lett. 60, 484 (1988).

[5] D. Ghoshal and A. Sen, Nucl. Phys. B584, 300 (2000).

[6] A. Sen, hep-th/9904207.

[7] A. Gerasimov and S. Shatashvili, J. High Energy Phys. 10 (2000) 034.

[8] E. Witten, Phys. Rev. D 46, 5467 (1992).

[9] S. Shatashvili, Phys. Lett. B 311, 83 (1993); Alg. Anal. 6, 215 (1994).

[10] D. Kutasov, M. Marino, and G. Moore, J. High Energy Phys. 10 (2000) 045.

[11] D. Ghoshal and A. Sen, J. High Energy Phys. 11 (2000) 021; D. Ghoshal, in Proceedings of Strings 2001, edited by A. Dabholkar et al. (American Mathematical Society, Providence, 2002), p. 107.

[12] D. Ghoshal, Reports No. MRI-P-0605001 and No. AEI2006-035.

[13] R. Rammal, G. Toulouse, and M. Virasoro, Rev. Mod. Phys. 58, 765 (1986).

[14] I. M. Gelfand, M. I. Graev, and I.I. Pitaetskii-Shapiro, Representation Theory and Automorphic Functions (Saunders, Philadelphia, 1969).

[15] N. Koblitz, p-Adic Numbers, p-adic Analysis and Zeta Functions, Graduate Texts in Mathematics Vol. 58 (Springer-Verlag, Berlin, 1977).

[16] F. Gouvêa, p-Adic Numbers: An Introduction (SpringerVerlag, Berlin, 1993).

[17] L. Brekke and P. Freund, Phys. Rep. 233, 1 (1993).

[18] I. V. Volovich, Classical Quantum Gravity 4, L83 (1987).

[19] B. Grossman, Phys. Lett. B 197, 101 (1987).

[20] D. Ghoshal, math-ph/0606003.

[21] B. Spokoiny, Phys. Lett. B 208, 401 (1988).

[22] D. Ghoshal, J. High Energy Phys. 09 (2004) 041.

[23] D. Ghoshal and T. Kawano, Nucl. Phys. B710, 577 (2005).

[24] P. Grange, Phys. Lett. B 616, 135 (2005).

[25] A. Zabrodin, Commun. Math. Phys. 123, 463 (1989).

[26] L. Chekhov, A. Mironov, and A. Zabrodin, Commun. Math. Phys. 125, 675 (1989).

[27] A. Comtet, S. Nechaev, and R. Voituriez, J. Stat. Phys. 102, 203 (2001); S. Nechaev and O. Vasilyev, J. Phys. A 37, 3783 (2004).

[28] C. Monthus and C. Texier, J. Phys. A 29, 2399 (1996).

[29] B. Dolan, W. Janke, D. Johnston, and M. Stathakopoulos, J. Phys. A 34, 6211 (2001).

[30] A. Zamolodchikov and Al. Zamolodchikov, hep-th/ 0101152 . 